University of Nebraska - Lincoln

DigitalCommons@University of Nebraska - Lincoln

\title{
4f hybridization and band dispersion in gadolinium thin films and compounds
}

Takashi Komesu

University of Nebraska-Lincoln, tkomesu2@unl.edu

H.-K. Jeong

University of Nebraska-Lincoln, hjeong@unl.edu

David Wooton

Air Force Institute of Technology, Wright Patterson Air Force Base, $\mathrm{OH}$

Yaroslav B. Losovyj

University of Nebraska-Lincoln, ylozovyy@indiana.edu

J. N. Crain

University of Wisconsin-Madison

See next page for additional authors

Follow this and additional works at: https://digitalcommons.unl.edu/physicsdowben

Part of the Physics Commons

Komesu, Takashi; Jeong, H.-K.; Wooton, David; Losovyj, Yaroslav B.; Crain, J. N.; Bissen, M.; Himpsel, F. J.; Petrosky, J. C.; Tang, Jinke; Wang, Wendong; Yakovkin, I. N.; and Dowben, Peter A., "4f hybridization and band dispersion in gadolinium thin films and compounds" (2009). Peter Dowben Publications. 238.

https://digitalcommons.unl.edu/physicsdowben/238

This Article is brought to you for free and open access by the Research Papers in Physics and Astronomy at DigitalCommons@University of Nebraska - Lincoln. It has been accepted for inclusion in Peter Dowben Publications by an authorized administrator of DigitalCommons@University of Nebraska - Lincoln. 


\section{Authors}

Takashi Komesu, H.-K. Jeong, David Wooton, Yaroslav B. Losovyj, J. N. Crain, M. Bissen, F. J. Himpsel, J. C. Petrosky, Jinke Tang, Wendong Wang, I. N. Yakovkin, and Peter A. Dowben 


\section{$4 \mathrm{f}$ hybridization and band dispersion in gadolinium thin films and compounds}

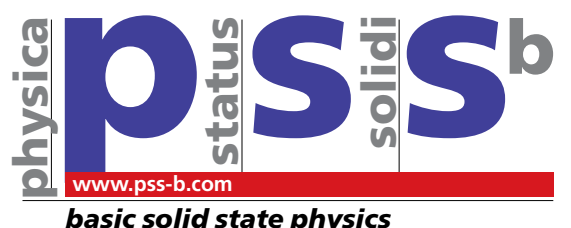

basic solid state physics

Takashi Komesu $^{1, a}$, H. K. Jeong ${ }^{1, b}$, David Wooton ${ }^{2}$, Ya. B. Losovyj ${ }^{1,3}$, J. N. Crain ${ }^{4}$, M. Bissen ${ }^{5}$, F. J. Himpsel ${ }^{4}$, J. Petrosky ${ }^{2}$, Jinke Tang ${ }^{6}$, Wendong Wang ${ }^{6}$, I. N. Yakovkin ${ }^{7}$, and P. A. Dowben ${ }^{1, *}$

${ }^{1}$ Department of Physics and Astronomy and the Nebraska Center for Materials and Nanoscience, University of Nebraska-Lincoln, P.O. Box 880111, Lincoln, NE 68588-0111, USA

${ }^{2}$ Air Force Institute of Technology, 2950 Hobson Way, Wright Patterson Air Force Base, OH 45433-7765, USA

${ }^{3}$ Center for Advanced Microstructures and Devices, Louisiana State University, 6980 Jefferson Highway, Baton Rouge,

LA 70806, USA

${ }^{4}$ Department of Physics, B317 Sterling Hall, University of Wisconsin-Madison, 1150 University Ave., Madison, WI 53706-1390, USA

${ }^{5}$ Synchrotron Radiation Center, 3731 Schneider Drive, Stoughton, WI 53589-3097, USA

${ }^{6}$ Department of Physics and Astronomy, University of Wyoming, Laramie, WY 82071, USA

${ }^{7}$ National Academy of Science of Ukraine, Institute of Physics, Prospect Nauki 46, 03028, Kiev, Ukraine

${ }^{a}$ Current address: SPring 8, RIKEN Harima Institute, Quantum Order Research Group, 1-1-1 Kouto, Sayo-cho,

Sayo-gun, Hyogo 679-5148, Japan

${ }^{\mathrm{b}}$ Current address: Sungkyunkwan University, Department of Physics, Center for Nanotubes and Nanostructured Composites, Suwon 440-746, Korea (ROK)

Received 18 August 2008, revised 18 December 2008, accepted 19 December 2008

Published online 3 April 2009

PACS 71.10.-w, 71.20.Eh, 71.45.Gm, 79.60.-i

* Corresponding author: e-mail pdowben@unl.edu, Phone: 1-402-472-9838, Fax: 1-402-472-2879

There is interplay between intra-atomic orbital hybridization and extra-atomic hybridization in various gadolinium systems, which affects magnetic coupling and electron itinerancy (localization). The results do not always follow expectation. The experimental band structure of thin $\mathrm{Gd}(0001)$ films, grown on the $\mathrm{Mo}(112)$ surface, along the $\bar{\Gamma}-\bar{M}$ does not agree with ex- pectations even qualitatively. In particular, the dispersion of the gadolinium band, with strong $5 \mathrm{~d}$ weight near $2 \mathrm{eV}$ binding energy provides considerable evidence to support the case for $4 \mathrm{f}-$ $5 \mathrm{~d}$ hybridization, with increasing $5 \mathrm{~d}$ localization. On the other hand, there is also evidence of extra-atomic $\mathrm{Gd} 4 \mathrm{f}$ hybridization leading band dispersion in the occupied $4 \mathrm{f}$ levels in $\mathrm{Gd}_{2} \mathrm{O}_{3}$.
In local moment $4 \mathrm{f}$ systems, two issues that have a strong influence on our understanding of exchange coupling are the extent of $4 \mathrm{f}$ localization or itinerancy AND the extent of $4 \mathrm{f}-5 \mathrm{~d}$ hybridization. While a number of experimental studies have been undertaken to investigate the spin-polarized band structure of strained $\operatorname{Gd}(0001)$ [1-4], interpretation of experimental data is complicated by the possibility of $4 \mathrm{f}-5 \mathrm{~d}$ hybridization. Singh [5] has suggested that there is hybridization with the upper part of the gadolinium 4f manifold (spin minority 4f) with the unoccupied $5 \mathrm{~d} / 6 \mathrm{~s}$ bands, and this is very consistent with the experimental band structure [4]. Here we address the issue of $4 f-5 d$ hybridization with respect to the spin majority $4 f^{7}$ gadolinium (lower part) manifold.
Without a treatment of the $4 \mathrm{f}$ electrons that includes hybridization, correlation energies, and band structure, the calculations (despite the diversity of approaches) have exhibited only limited agreement with photoemission experiments [4]. In the case of strained Gd(0001), comparisons of the occupied band structure with theory are dramatically worse than for strain relieved $\mathrm{Gd}(0001)$ grown on W(110) $[4,6,7]$.

It has been established that the local spin density approximation (LSDA) fails to describe correctly electronic structure of strongly correlated systems such as the $4 \mathrm{f}$ metals $[5,8,9]$. The inability of LSDA to correctly describe $4 \mathrm{f}$ metals appears to occur mainly because of the incorrect position of spin up $\mathrm{f} \uparrow$ and spin down $\mathrm{f} \downarrow$ bands if the $4 \mathrm{f}$ elec- 
trons are treated as bands and not as core states [10-12]. Harmon and Freeman [13] calculated the band structure for Gd by APW method using the exchange-correlation $\mathrm{X}_{\alpha}$ potential with two values i.e. $\alpha=1$ ("total Slater's exchange") and $\alpha=2 / 3$ ("Kohn-Sham potential"). The occupied $4 \mathrm{f} \uparrow$ states were treated as core states and given a rigid value of magnetic moment $\mu=7 \mu_{\mathrm{B}}$ associated with spin-up $4 \mathrm{f}^{7}$ electrons. In order to get the experimental value of $7.55 \mu_{\mathrm{B}}$ for $\mathrm{Gd}$, the rest magnetic moment, some 0.55 $\mu_{\mathrm{B}}$, was provided by properly choosing the configuration of the $5 \mathrm{~d} 6 \mathrm{~s}$ valence electrons. The results for $\alpha=1$ were in reasonable agreement with experiment, though the obtained width of the valence band (from the bottom at $\Gamma$ to $\mathrm{E}_{\mathrm{F}}$ ) of $3.1 \mathrm{eV}$ seems too small compared to the values derived from photoemission of about $4 \mathrm{eV}$.

Harmon et al. [9] and Singh [5] suggested that correct total energies for $\mathrm{Gd}$ could not be obtained if $4 \mathrm{f}$ electrons were treated as core states. The increasingly compelling evidence that gadolinium was a local moment system, with correlated electrons [7], provoked the development of an improved version of LSDA by including the Hubbard U, taken from the mean-field approach [8]. The LSDA + U method has proved fruitful in recent calculations of the bulk and surface electronic structure of relatively unstrained Gd [14-16]. Due to a better accounting of the intra-atomic correlations, the $4 \mathrm{f}$ minority band rises away from Fermi level while the $4 \mathrm{f}$ majority band increases in binding energy to the correct position [14] (approximately $-8.5 \mathrm{eV}$ with respect to $\mathrm{E}_{\mathrm{F}}$ [7]). Wu et al. [17], using von Barth and Hedin's exchange-correlation potential, calculated the $\mathrm{Gd}(0001)$ band structure by the FLAPW method. The majority $4 \mathrm{f}^{7}$ electrons were treated as core states. Despite using an occupied s-d band width (almost $6 \mathrm{eV}$ ) that is too large, the results are in qualitative agreement with angle-resolved photoemission [18-20] and inverse photoemission [21-23] data, again of the relatively unstrained $\operatorname{Gd}(0001)$. In particular, the calculations reveal the existence of the spin majority (in vicinity of $\bar{\Gamma}$ ) surface band of $\mathrm{d}_{3 z^{2}-r^{2}}$ symmetry $0.3 \mathrm{eV}$ below $\mathrm{E}_{\mathrm{F}}[4,17,19]$ as well as spin minority surface resonance bands above $\mathrm{E}_{\mathrm{F}}$ which are observed in the spin-polarized photoemission $[2,24]$ and spin-polarized inverse photoemission [22, 23] spectra respectively. The issue of correct description of 4f states was also addressed by Eriksson et al. [12] where it was suggested that the "first-principle" (pure) LSDA approach could be resuscitated, provided that the $4 \mathrm{f}$ states are treated as core states.

Following Bylander and Kleinman [10], Yakovkin et al. [4] adopted the parameter description for correlation energy, while the core electrons are treated within the same approximation for exchange-correlation potential.

With expansive strain, increased localization will increase the $5 \mathrm{~d}$ band localization and might well result in greater intra-atomic overlap between $4 \mathrm{f}$ and $5 \mathrm{~d}$ levels [6]. This, in-turn, should also affect the bottom of the $5 \mathrm{~d} / 6 \mathrm{~s}$ bulk bands as indicated by angle resolved photoemission band mapping, reported elsewhere [4,6]. Reasonable agreement between experiment and theory was realized excepting the highest binding energy $5 \mathrm{~d} / 6 \mathrm{~s}$ Gd bulk band - the occupied bulk band most likely to provide indications of occupied $4 \mathrm{f}-5 \mathrm{~d}$ hybridization. If this is indeed the case, then it is questionable whether the $\mathrm{Gd} 4 \mathrm{f}$ levels can be treated as pure core states. If the $4 \mathrm{f}$ levels cannot be treated as shallow core level states, then a proper treatment of the $4 \mathrm{f}$ levels needs to be revisited.

The possible effect of strain on $\mathbf{G d} \mathbf{5 d}$ localization: The expansive $4 \%$ in-plane strained $\mathrm{Gd}(0001)$ on $\operatorname{Mo}(112)$ results in a quite different electronic structure and altered magnetic properties compared to the strain relieved $\mathrm{Gd}(0001)$ grown on W(110) [25], as has been noted elsewhere $[2,4,6,26]$. Based on studies of rare earth alloys, Andrianov [27-29] suggested that the Fermi surface is sensitive to both composition and lattice deformation. $\operatorname{Gd}(0001)$ grown on $\mathrm{Mo}(112)$ exhibit expansive strain and an increased in-plane lattice constant, expanded by $4 \%$ for a film thickness of 30 to $150 \AA$ [6]. Several interesting characteristics of such strained Gd films have already been noted: large magneto-elastic interactions [30,31], enhance Curie temperatures [1], a different electronic band structure compared to that of bulk "unstrained" Gd [2-6], and others as described below. Such experimental observations have also been noted for other rare earths as well [34-36].

In comparing the calculated electronic band structures of bulk "unstrained" Gd and the strained Gd film, two significant differences emerge in both the calculations of Yakovkin [4] and Freeman [37] as compared with experiment. As schematically shown in Fig. 1, the largest binding energy $5 \mathrm{~d} / 6 \mathrm{~s}$ bulk band exhibits less dispersion with increasing expansive strain. The Gd slab calculations result in narrower occupied band (smaller dispersion) with increasing lattice constant, yet the direction of the dispersion remains the same. In addition, regardless of strain, the band is $\Delta_{1}$ or $\alpha_{1}$ symmetry $\left(5 \mathrm{~d}_{z^{2}-r^{2}}, 6 \mathrm{~s}\right.$ character), even with extensive expansive strain.

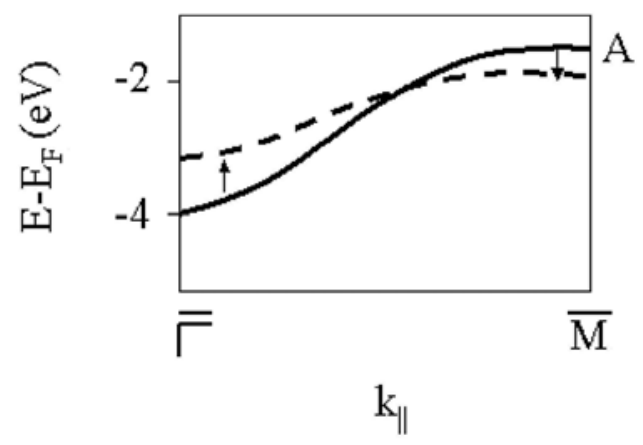

Figure 1 This is a schematic band structure showing the dispersions of the highest binding energy $5 \mathrm{~d}$ bulk bands. Solid line represents the unstrained $\mathrm{Gd}(0001)$ and dashed line is for the expansively strained Gd. 
These expectations from theory are not reproduced in experiment [6]. These bulk bands are experimentally observed to change symmetry from $\Delta_{1}, \Delta_{2}\left(5 \mathrm{~d}_{z^{2}-r^{2}}, 6 \mathrm{~s}\right)$ for unstrained $\mathrm{Gd}(0001)$ on $\mathrm{W}(110)$ to $\Delta_{5}, \Delta_{6}$ at $\bar{\Gamma}$ with the $4 \%$ expansive strain [6]. The second difference between experiment and theory is the band dispersion of the highest binding energy bulk bands, discussed herein.

We have mapped out band structure of thin Gd films grown on the Mo (112) surface along the $\bar{\Gamma}-\bar{M}$ highsymmetry direction of the surface Brillouin zone. The experiments were carried out in an UHV chamber equipped with a Scienta 200 electron analyzer, at the Synchrotron Radiation Center in Stoughton, Wisconsin, with considerable wave vector and energy resolution in angle-resolved photoemission. The clean Mo (112) surface was obtained after the standard surface preparation treatment [6], which included heating in oxygen atmosphere with subsequent serial flashing to the temperatures above $2100 \mathrm{~K}$. The Gd (0001) films were grown at room temperature at the base pressure of $1 \times 10^{-10}$ Torr and subsequently annealed. The crystal of quality of the $\mathrm{Gd}$ (0001) films was determined by LEED, which was also used to determine the extent of the expansive strain, along with the critical points in the experimental band mapping. Spectra from the photoemission are also used to confirm crystal quality since contaminated spectra are definitely different $[6,7]$. The in-plane lattice constant is expanded by $4 \%$ compared to that of the bulk $\operatorname{Gd}(a=3.6 \AA)$.

Angle-resolved spectra were taken at around $200 \mathrm{~K}$ and the spin-integrated wave vector spectral density is plotted in Fig. 2. The spin integrated data can be easily compared with the spin polarized band mapping [2-4]. The slight displacement in the wave vector dependent binding energies between spin-polarized data and the spin integrated data are due to changes in $k_{\perp}$ (the spin integrated spectral density data were taken at $32 \mathrm{eV}$ while the spin resolved band mapping was acquired at $35 \mathrm{eV}$ ) affecting principally the bulk bands. In spite of the difference in photon energy between the two sets of data, the spin majority (blue) and spin minority (red) bands agree relatively well with the wave vector dependent spectral density from present angleresolved and spin-integrated spectra.

While the bands around the Fermi energy are described very well elsewhere [2-4], a repetition of this is beyond the scope of the present paper. The bulk 5d6s bands (labeled A and B in Fig. 2) do not show the expected positive slope in the dispersion compared to unstrained Gd due to the strain and the second bulk band $\mathrm{B}$, nicely resolved away from $\bar{\Gamma}$, is not expected from theory at all. This band is a result of strain but the origin of this is more difficult to explain.

Theory, in which the $4 \mathrm{f}$ states are treated as core states or a perturbation to the valence band structure could be deficient. The bulk bands we label the 'A' and ' $B$ ' bands may arise from combination of $4 \mathrm{f}$ and $5 \mathrm{~d}$ wave functions. We would expect more weight of $5 \mathrm{~d}$ states near $\bar{\Gamma}$ and more 4f states near $\bar{M}$. This profound disagreement between the-

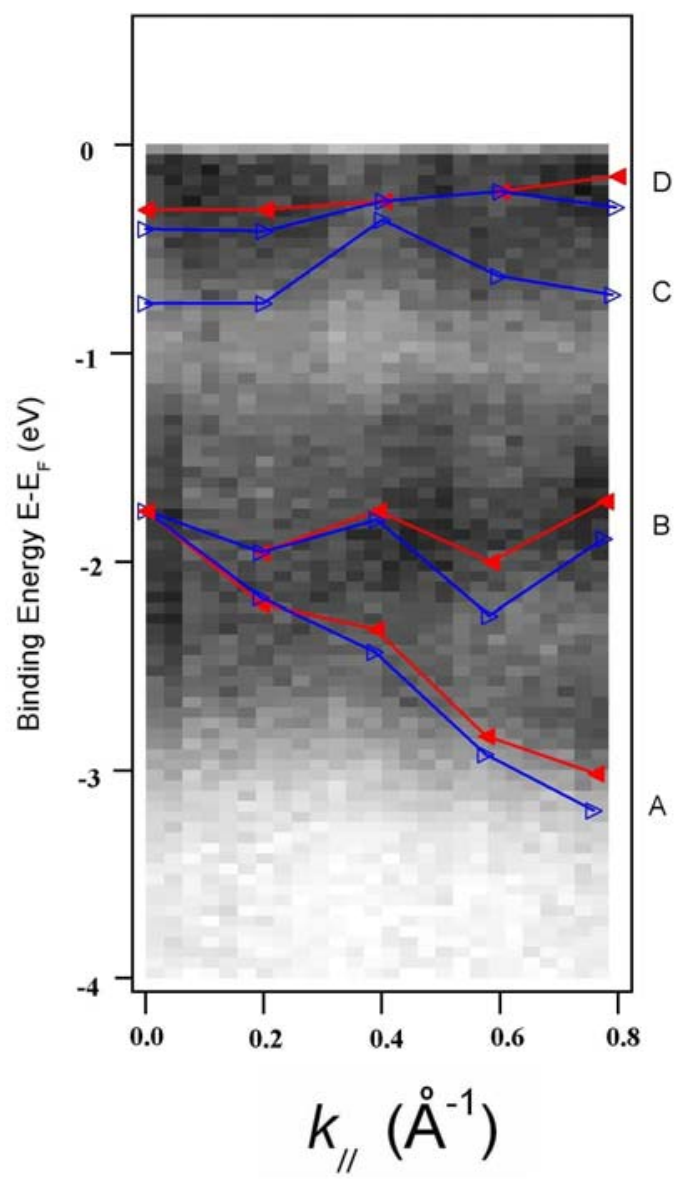

Figure 2 Spin-integrated Scienta data at $32 \mathrm{eV}$, with an overlay of the band dispersion inferred from spin resolved photoemission taken at a photon energy of $35 \mathrm{eV}$, and select wave vectors. The latter data is adapted from reference [2,3]. In the spin resolved data overlay, red triangles are spin minority and blue is spin majority. Higher photoemission intensities are shown dark.

ory and experiments we take to be an indication of $4 f-5 d$ mixing.

While considerable agreement with experiment was obtained [4], there are two significant differences between theory and the experimental band mappings both in regard to the strained Gd films the $5 \mathrm{~d}, 6$ s occupied bulk spin majority and minority subbands found at approximately 1.8 $\mathrm{eV}$ binding energy (at $35 \mathrm{eV}$ photon energy) to $2.0 \mathrm{eV}$ binding (at $32 \mathrm{eV}$ photon energy) at $\bar{\Gamma}$. Light polarization photoemission experiments indicate that these bulk bands change symmetry from $\Delta_{1}, \Delta_{2}\left(5 \mathrm{~d}_{z^{2}-r^{2}} 6 \mathrm{~s}\right)$ for unstrained $\mathrm{Gd}(0001)$ on W(110) to $\Delta_{5}, \Delta_{6}$ at $\bar{\Gamma}$ with the $4 \%$ expansive strain for $\mathrm{Gd}(0001)$ grown on $\mathrm{Mo}(112)$ [6]. No matter how extensive the strain, expansive strain does not result in a change in symmetry of these bands from $\Delta_{1}, \Delta_{2}\left(5 \mathrm{~d}_{z^{2}-r^{2}}\right.$ 6s).

Since strained Gd film has a greater lattice constant, owing to the slight expansive strain (4\%) compared to Gd 


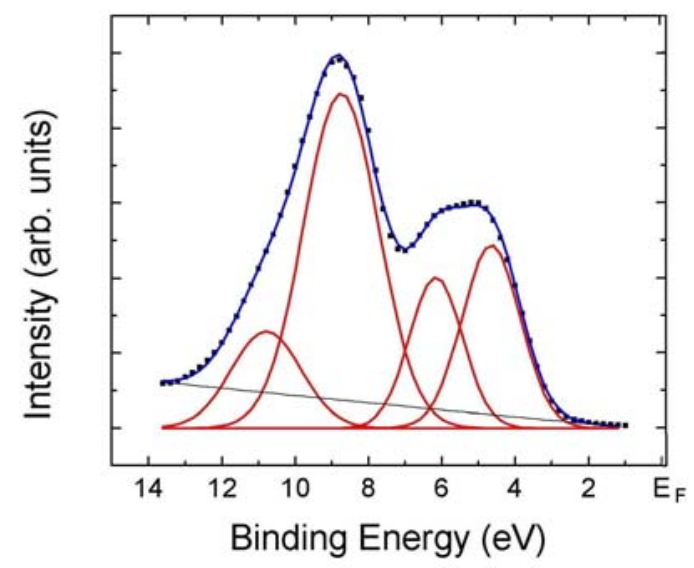

Figure 3 The photoemission spectrum of the valence band for $\mathrm{Gd}_{2} \mathrm{O}_{3}(-402)$. The various components contributing to the valence band structure are indicated. The photon energy is $100 \mathrm{eV}$ and the light incidence angle is $45^{\circ}$. All photoelectrons were collected along the surface normal at $T=240^{\circ} \mathrm{C}$. Binding energies are in $E_{\mathrm{F}}-E$.

bulk, adjacent Gd atomic wave functions decrease in the extent of overlap. This, in turn, could increase the localization of the $\mathrm{Gd} 5 \mathrm{~d}$ band and thus increase the $5 \mathrm{~d}-4 \mathrm{f}$ wave function overlap and the potential for intra-atomic hybridization. $5 \mathrm{~d}$ band overlap may be a key to $4 \mathrm{f} / 5 \mathrm{~d}$ hybridization. In the more localized rare earth compounds, Olson et al. [32] found evidence for $4 \mathrm{f}$ delocalization in $\mathrm{CeSb}_{2}$, in the form of dispersing Bloch states. Of course $f$ delocalization can also occur, but is more expected for the $5 \mathrm{f}$ levels. Joyce et al. [33] also found clear evidence of similar dispersion in the uranium $5 \mathrm{f}$ levels. In some sense, the result here are similar to the apparent effects of $4 f-5 d$ hybridization suggested by the result seen for $\operatorname{ErAs}(100)$, where strong $4 \mathrm{f}$ intra-atomic hybridization was implicated, and a direct confirmation of an occupied $4 \mathrm{f}$ band structure inferred from the data but could not be established [38,39].

Gd 4 f band structure: In the $\mathrm{Gd}^{3+}$ and mixed valence systems, Gd $4 \mathrm{f}$ hybridization with nearest neighbor atoms is expected $[40,41]$ and observed. To actually demonstrate this, we have mapped out the bulk band structure of the Gd $4 \mathrm{f}$ weighted bands in a system where the Gd 5d/6s bands are heavily suppressed: $\mathrm{Gd}_{2} \mathrm{O}_{3}$. This experimental band mapping was done along the normal to $(-402)$, using angle-resolved photoemission. Here, experiments were performed using the $3 \mathrm{~m}$ toroidal grating monochromator $(3$ $\mathrm{m}$ TGM) beam line [42] in a UHV chamber previously described $[38,39,42]$. The Fermi level $\left(E_{F}\right)$ was established from a gold film in electrical contact with the sample and measurements were carried out at ambient temperatures.

When $\mathrm{Gd}_{2} \mathrm{O}_{3}$ is grown on $\mathrm{Si}(100)$ using a pure $\mathrm{Gd}_{2} \mathrm{O}_{3}$ target by pulsed laser deposition, the substrate has a strong influence on the texture and crystal structure of the $\mathrm{Gd}_{2} \mathrm{O}_{3}$ film [43]. Such films are highly textured monoclinic $\mathrm{Gd}_{2} \mathrm{O}_{3}$, not the more expected cubic structure [44-46]. The textured structure is such that the $(-402)$ planes mostly lie along the surface of the film [43]. The lattice spacing along the $\langle-402\rangle$ direction is about $0.2965 \mathrm{~nm}$ so the repeat along the surface normal is eight times the $(-402)$ layer spacing or about $2.372 \mathrm{~nm}$. So while the $\mathrm{Gd}_{2} \mathrm{O}_{3}$ unit cell is large with inequivalent $\mathrm{Gd}$ (three) and oxygen atoms (in a number of inequivalent sites), it is a system in which some $4 \mathrm{f}$ band dispersion might be observed, in spite of some 84 gadolinium bands per unit cell.

The valence band density of states, as determined by photoemission for $\mathrm{Gd}_{2} \mathrm{O}_{3}$, are very similar to that observed for $3 \% \mathrm{Gd}$-doped hafnium oxide films $[41,42,47]$ and reported elsewhere for $\mathrm{Gd}_{2} \mathrm{O}_{3}$ [48,49], as indicated in Fig. 3. The major difference is that for $\mathrm{Gd}_{2} \mathrm{O}_{3}$, we require 4 components at a minimum to fit the valence band spectra, as also indicated in Fig. 3, as opposed to three components needed for $\mathrm{Gd}$ doped $\mathrm{HfO}_{2}$, in the monoclinic phase [41, 42,47]. Confirmation that the Gd $4 \mathrm{f}$ states contribute to the photoemission components at about 8.7 to $9.5 \mathrm{eV}$ and the 'shoulder' at 10 to $11 \mathrm{eV}$ binding energies is found in resonant photoemission (i.e., constant initial state spectroscopy) measurements of $\mathrm{Gd}$ doped $\mathrm{HfO}_{2}[42,47]$. The photoelectron intensities from $\mathrm{Gd}$ doped $\mathrm{HfO}_{2}$, determined from the feature at about $9.5 \mathrm{eV}$ binding energy (from the Fermi level) is strongly enhanced at about $152 \mathrm{eV}$, or at the $4 \mathrm{~d}^{10} 4 \mathrm{f}^{7} \rightarrow 4 \mathrm{~d}^{9} 4 \mathrm{f}^{8} \rightarrow 4 \mathrm{~d}^{10} 4 \mathrm{f}^{6}+\mathrm{e}^{-}$super Coster-Kronig transition $[40,41,41,50,51]$, leading to a classic Fano resonance.

Since the two components, identified as heavily Gd $4 \mathrm{f}$ in weight, are separated by slightly more than $2 \mathrm{eV}$, we must conclude that the higher binding energy component is, in fact, a consequence of the surface to bulk shift, and is the surface component. The Gd occupied $4 \mathrm{f}$ surface-tobulk shift is only about $0.4 \mathrm{eV}$ for $\mathrm{Gd}$ metal, but is expected to be much larger with oxidation $[49,52]$, with the surface component at the greater binding energy. It is the larger intensity component, at 8.7 to $9.5 \mathrm{eV}$ binding energy, associated with the bulk $\mathrm{Gd} 4 \mathrm{f}$ weighted bands of $\mathrm{Gd}_{2} \mathrm{O}_{3}$, that are of interest in establishing the $\mathrm{Gd} 4 \mathrm{f}$ band structure.

Because of the high degree of order and the strong texture growth along $\langle-402\rangle$, we have been able to plot out the bulk band structure along $\langle-402\rangle$. Because the photoelectrons are collected along the surface normal in the sequence of photon energy dependent photoemission spectra, the binding energy shifts of many of the observed photoemission features is indicative of band dispersion along the electron wave vector normal to the surface, $k_{\perp}$. The value of $k_{\perp}$ can be estimated from the photoelectron kinetic energy making some assumptions about the inner potential $U_{\text {in }}$ :

$$
k_{\perp}=\sqrt{\left.\frac{2 m}{\hbar^{2}}\left\{E_{\mathrm{kin}}(\cos (\theta))^{2}+U_{\mathrm{in}}\right)\right\}} .
$$




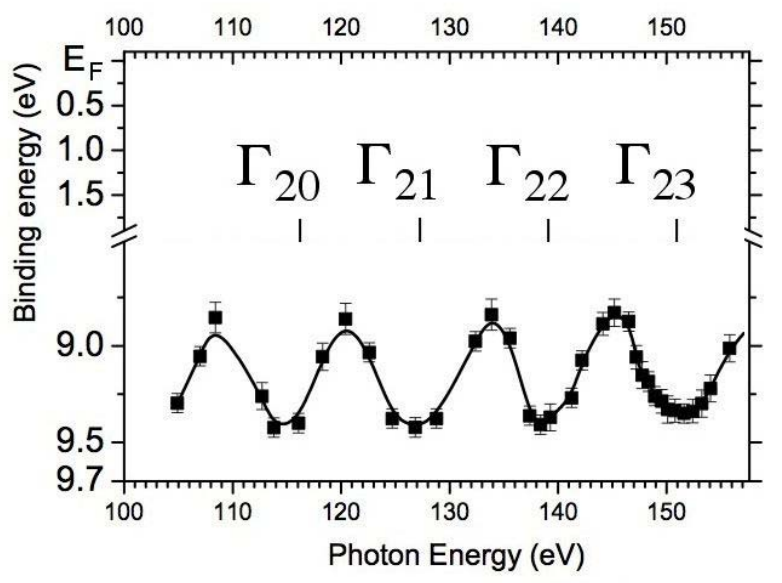

Figure 4 The dispersion of the Gd $4 \mathrm{f}$ component of the valence band of gadolinium oxide, with changing photon energy. The critical points are indicated, assuming no inner potential and the predicted lattice spacing of $23.7 \AA$. Binding energies are in $E_{\mathrm{F}}-E$.

The dispersion of the component we attribute to the $\mathrm{Gd}$ $4 \mathrm{f}$ dependence level at 9 to $9.5 \mathrm{eV}$ binding energies is summarized in Fig. 4. The dependence on photon energy shows band critical points repetition suggesting periodicity of about $22 \pm 2 \AA$ perpendicular to the film or along the surface normal, close to the value of $23 \AA$ as expected from the crystallography.

For a dielectric insulator we would expect that the value of the inner potential to be effectively negligible, as seems to be the case here. We cannot establish that the inner potential is zero, but from our measurements that establishes the critical points for the 19th through to the 23rd Brillouin zone, the inner potential is quite small and is no more than 1-2 eV, at most. Assuming a value of zero for the inner potential, and a lattice constant of $23.72 \AA$, the calculated critical points are in good agreement with the experimental band structure, as indicated in Fig. 4.

What seems clear is that not only do we have strong Gd 4f hybridization with the oxygen for $\mathrm{Gd}_{2} \mathrm{O}_{3}$ and we have a small amount of band dispersion in the band strongly weighted with a $\mathrm{Gd} 4 \mathrm{f}$ contribution for $\mathrm{Gd}_{2} \mathrm{O}_{3}$. The band dispersion is small (about $500 \mathrm{meV}$ ), but evident nonetheless. The $\mathrm{Gd} 4 \mathrm{f}$ weighted band is placed well away from the Fermi level in the valence band of $\mathrm{Gd}_{2} \mathrm{O}_{3}$ but still exhibits band structure.

Summary: We have mapped out band structure of thin Gd films grown on the Mo (112) surface along the $\bar{\Gamma}-\bar{M}$ and the results are largely consistent with previous work [1-4]. Nonetheless, strong deviations from theory are reproduced, and this in turn implicates $4 \mathrm{f}-5 \mathrm{~d}$ hybridization. This 4f-5d hybridization appear now not only to occur for the unoccupied band structure $[4,5]$ but may exist for the occupied Gd 4f band structure as well. The hybridization for the unoccupied band structure results from the energy position (overlap) of the $d-f$ bands, which is not so very evident for the occupied bands. In spite of the high index face adopted by $\mathrm{Gd}_{2} \mathrm{O}_{3}$ thin films on $\mathrm{Si}(100)$, and the large unit cell of $\mathrm{Gd}_{2} \mathrm{O}_{3}, \mathrm{Gd} 4 \mathrm{f}$ band structure is nonetheless and perhaps surprisingly apparent for $\mathrm{Gd}_{2} \mathrm{O}_{3}$.

This raises some interesting issues that have not yet been successfully addressed in theory. First, how can we account for the observed change in symmetry from $\Delta_{1}, \Delta_{2}$ $\left(5 \mathrm{~d}_{z^{2}-r^{2}}, 6 \mathrm{~s}\right)$ for unstrained $\mathrm{Gd}(0001)$ on $\mathrm{W}(110)$ to $\Delta_{5}, \Delta_{6}$ at $\bar{\Gamma}[6]$ and the resulting influence of the slope of the band dispersion. Further, does this change in valence band symmetry indeed imply intra-atomic Gd $4 \mathrm{f}-5 \mathrm{~d}$ hybridization? Second, are the Gd 4 f levels really shallow core levels, or states with a weak band dispersion?

Acknowledgements The authors acknowledge insightful discussions with B. Harmon, Dongqi Li, and A. Petukov. The authors would like to thank Carlo Waldfried for his technical help with some of the measurements. This work was supported by the Office of Naval Research (Grant No. N00014-06-1-0616), the Defense Threat Reduction Agency (Grant No. HDTRA1-07-1-0008 and MIPR 06-2310M), and the Nebraska Research Initiative. This work was undertaken in partial fulfillment of the degree at AFIT by one author (DW). The views expressed in this article are those of the authors and do not reflect the official policy or position of the Air Force, Department of Defense or the U.S. Government. Part of this work was performed at the Synchrotron Radiation Center (SRC) of the UW-Madison, which is supported by NSF Grant \#DMR-0537588; the Center of Advance Microstructures and Devices is supported by the Louisiana Board of Regents.

\section{References}

[1] C. Waldfried, T. McAvoy, D. Welipitiya, P.A. Dowben, and E. Vescovo, Europhys. Lett. 42, 685 (1998).

[2] C. Waldfried, T. McAvoy, D. Welipitiya, T. Komesu, P.A. Dowben, and E. Vescovo, Phys. Rev. B 58, 7434 (1999).

[3] Takashi Komesu, C. Waldfried, and P.A. Dowben, Phys. Lett. A 256, 81 (1999).

[4] I.N. Yakovkin, T. Komesu, and P.A. Dowben, Phys. Rev. B 66, 035406 (2002).

[5] D.J. Singh, Phys. Rev. B 44, 7451 (1991).

[6] C. Waldfried, D. N. McIlroy and P.A. Dowben, J. Phys.: Condens. Matter 9, 10615 (1997).

[7] P.A. Dowben, D.N. McIlroy and Dongqi Li, Handbook on the Physics and Chemistry of Rare Earths, Edited by K.A. Gschneidner and LeRoy Eyring, North Holland Press, vol. 24, chapter 159 (1997) 1-46; Magnetism and Electronic Correlations in Local Moment Systems, Edited by M. Donath, P.A. Dowben and W. Nolting, World Scientific (1998) ISBN 981-02-3538-0.

[8] V.I. Anisimov, J. Zaanen, and O.K. Andersen, Phys. Rev. B 44, 943 (1991).

[9] B.N. Harmon, V.P. Antropov, A.I. Liechtenstein, I.V. Solovyev, and V.I. Anisimov, J. Phys. Chem. Solids 56, 1521 (1995).

[10] D.M. Bylander and L. Kleinman, Phys. Rev. B 50, 1363 (1994). 
[11] D.M. Bylander and L. Kleinman, Phys. Rev. B 49, 1608 (1994); J. Morrison, D.M. Bylander, and L. Kleinman, Phys. Rev. Lett. 71, 1083 (1993).

[12] O. Eriksson, R. Ahuja, A.Ormeci, J. Tregg, O. Hjorstam, P. Soderlind, B. Johansson, and J.M. Willis, Phys. Rev. B 52, 4420 (1995).

[13] B.N. Harmon and A.J. Freeman, Phys. Rev. B 10, 1979 (1974).

[14] R.F. Sabirianov and S.S. Jaswal, Phys. Rev. B 55, 4117 (1997).

[15] A.B. Shick, A.I. Liechtenstein, and W.E. Pickett, Phys. Rev. B 60, 10763 (1999).

[16] A.B. Shick, W.E. Pickett, and C.S. Fadley, Phys. Rev. B 61, R9213 (2000).

[17] R. Wu, C. Li, A.J. Freeman, and C.L. Fu, Phys. Rev. B 44, 9400 (1991).

[18] Dongqi Li, C.W. Hutchings, P.A. Dowben, C. Hwang, RongTzong Wu, M. Onellion, A.B. Andrews, and J.L. Erskine, J. Magn. Magn. Mater. 99, 85 (1991).

[19] R.q. Wu and A.J. Freeman, J. Magn. Magn. Mater. 99, 81 (1991).

[20] D. Li, J. Zhang, P.A. Dowben and M. Onellion, Phys. Rev. B 45, 7272 (1992).

[21] Dongqi Li, P.A. Dowben, J.E. Ortega, and F.J. Himpsel, Phys. Rev. B 49, 7734 (1994).

[22] M. Donath, B. Gubanka, and F. Passek, Phys. Rev. Lett. 77, 5138 (1996)

[23] M. Donath, J. Phys.: Condens. Matter 11, 9421 (1999).

[24] Dongqi Li, J. Pearson, S.D. Bader, D.N. McIlroy, C. Waldfried, and P.A. Dowben, Phys. Rev. B 51, 13895 (1995).

[25] D. Weller and S.F. Alvarado, J. Appl. Phys. 59, 2908 (1986).

[26] T. Komesu, C. Waldfried, and P.A. Dowben, IEEE Trans. Magn. 36, 2915 (2000).

[27] A. Andrianov, JEPT Lett. 55, 666 (1992).

[28] A. Andrianov, J. Magn. Magn. Mater. 140-144, 749 (1995).

[29] A.V. Andrianov, D.I. Kosarev, and A.I. Beskrovnyi, Phys. Rev. B 62, 13844 (2000).

[30] R.G. Chapman and N.H. March, J. Magn. Magn. Mater. 61, 81 (1986).

[31] S. Legvold, B.N. Harmon, B.J. Beaudry, P. Burgart, D.R. Younkin, and H.W. White, Phys. Rev. B 16, 4986 (1977).

[32] C.G. Olson, S.J. Chase, P. Canfield, and D.W. Lynch, J. Electron Spectrosc. Relat. Phenom. 93, 175 (1998).

[33] A.B. Andrews, J.J. Joyce, A.J. Arko, J.D. Thompson, J. Tang, J.M. Lawrence, and J.C. Hemminger, Phys. Rev. B 51, R3277 (1995).

[34] A.V. Andrianov, D.I. Kosarev, and A.I. Beskrovnyi, Phys. Rev. B 62, 13844 (2000).

[35] A.V. Andrianov, J. Magn. Magn. Mater. 197, 714-715 (1999).
[36] S.B. Palmer, G.J. McIntyre, A.V. Andrianov, R.J. Melville, J. Magn. Magn. Mater. 177, 1023 (1998).

[37] S.C. Hong and A.J. Freeman, Bull. Am. Phys. Soc. 42, 201 (1997).

[38] T. Komesu, H.-K. Jeong, J. Choi, C.N. Borca, P.A. Dowben, A.G. Petukhov, B.D. Schultz, and C.J. Palmstrøm, Phys. Rev. B 67, 035104 (2003).

[39] Chun-gang Duan, T. Komesu, H.-K. Jeong, C.N. Borca, Wei-Guo Yin, Jianjun Liu, W. N. Mei, P.A. Dowben, A.G. Petukhov, B.D. Schultz, and C.J. Palmstrøm, Surf. Rev. Lett. 11, 531 (2004).

[40] R. F. Sabirianov, W. N. Mei, Jing Lu, Y. Gao, X. C. Zeng, R.D. Bolskar, P. Jeppson, Ning Wu, A.N. Caruso, and P. A. Dowben, J. Phys.: Condens. Matter 19, 082201 (2007).

[41] Ya. B. Losovyj, I. Ketsman, A. Sokolov, K. D. Belashchenko, P.A. Dowben, J. Tang, and Z. Wang, Appl. Phys. Lett. 91, 132908 (2007).

[42] Y. Losovyj, I. Ketsman, E. Morikawa, Z. Wang, J. Tang, and P. Dowben, Nucl. Instrum. Methods Phys. Res. A 582, 264 (2007).

[43] Ya. B. Losovyj, D. Wooten, J. Colon-Santana, J.-H. Michael An, K. Belashchenko, N. Lozova, J. Petrosky, A. Sokolov, J. Tang, W. Wang, N. Arulsamy, and P.A. Dowben, J. Phys.: Condens. Matter 21, 045602 (2009).

[44] G. Haire and L. Eyring, Handbook on the Physics and Chemistry of Rare Earths, vol. 18 (North-Holland, Amsterdam, 1994), p. 429.

[45] N. Horosaki, S. Ogata, and C. Kocer, J. Alloys Compd. 351, 31 (2003)

[46] B. P. Gila, K. N. Lee, W. Johnson, F. Ren, C. R. Abemathy, S. J. Pearton, M. Hong, J. Kwo, J. P. Mannaerts, and K. A. Anselm, IEEE Trans. IV-1, 182 (2000).

[47] I. Ketsman, Ya. B. Losovyj, A. Sokolov, J. Tang, Z. Wang, K. D. Belashchenko and P. A. Dowben, Appl. Phys. A 89, 489 (2007).

[48] Robert Meier, "Adsorption von Sauerstoff auf Lanthanidmetallen", Dissertation, Fachbereich Physik, Freie Universität Berlin (1999), p. 61.

[49] C. Schussler-Langeheine, R. Meier, H. Ott, Z. Hu, C. Mazumdar, A. Y. Grigoriev, G. Kaindl, and E. Weschke, Phys. Rev. B 60, 3449-3452 (1999).

[50] P.A. Dowben, Dongqi Li, Jiandi Zhang, and M. Onellion, J. Vac. Sci. Technol. A 13, 1549 (1995).

[51] T. Kachel, R. Rochow, W. Gudat, R. Jungblut, O. Rader, and C. Cabone, Phys. Rev. B 45, 7276 (1992).

[52] D. N. McIlroy, C. Waldfried, D. Li, J. Pearson, S. D. Bader, D.-J. Huang, P.D. Johnson, R.F. Sabiryanov, S. S. Jaswal, and P.A. Dowben, Phys. Rev. Lett. 76, 2802 (1996). 